\title{
White blood count and infarct size, myocardial salvage and clinical outcomes: the role of differentials
}

\author{
Nicholas G. Kounis • George D. Soufras • \\ Grigorios Tsigkas · George Hahalis
}

Received: 26 December 2013/Accepted: 30 December 2013/Published online: 3 January 2014

(C) Springer Science+Business Media Dordrecht 2014

Evidence suggests that inflammation, both local and systemic, plays an important role in the pathogenesis of acute myocardial infarction [1]. There is also evidence that increased concentrations of inflammatory markers such as C-reactive protein interleukin-2 and tumor necrosis factor can identify patients at high risk of future ischemic events, suggesting that the intensity of the inflammatory response influences clinical outcome in acute coronary syndromes [2, 3]. Multiple studies have demonstrated also an association between elevated leucocyte count, which is a non-specific marker of inflammation, and the extent of coronary artery disease or increased short-term or long-term risk of death in patients with various manifestations of coronary artery disease $[4,5]$. Other studies [6] have shown that total leucocyte count, but not C-reactive protein, predicts 1-year mortality in patients with acute coronary syndromes treated with percutaneous coronary intervention. Furthermore, recent studies have emphasized the usefulness of neutrophil to lymphocyte ratio in predicting short-and long-term mortality, thrombus formation, infarct related artery patency, TIMI flow grade after primary coronary angioplasty, impaired myocardial perfusion, ventricular arrhythmias and critical limb ischemia [7-9]. Indeed, in patients with non-ST-elevation myocardial infarction a ratio above 4.7 has been reported to be an independent predictor of short-term and long-term mortality [7] and ratio $>3.3$ was found to be an independent predictor of impaired coronary flow after primary percutaneous

\section{N. G. Kounis ( $\square)$}

Medical Sciences, Patras Highest Institute of Education

and Technology, Patras, Achaia, Greece

e-mail: ngkounis@otenet.gr

G. D. Soufras · G. Tsigkas · G. Hahalis

Departments of Cardiology, University of Patras Medical

School, Patras, Achaia, Greece coronary intervention and of in-hospital major adverse cardiac events in patients with ST-segment elevation myocardial infarction [10].

In a recent issue of the Int J Cardiovasc Imaging [11] and additional very interesting paper was published which shows that in patients undergoing primary percutaneous coronary intervention for ST-segment elevation myocardial infarction an elevated baseline white blood count is associated with less salvaged myocardium, larger infarct size and poorer clinical outcomes. All these papers and results really suggest a key role of inflammation in coronary artery disease. However, differential count was not available in these studies, and therefore, assessing the relative impact of white blood count subpopulations on myocardial infarction was not reported. Total white blood count comprises several cell types such as monocytes, lymphocytes and eosinophils all of which are implicated in the development, progression and instability of atherosclerotic plaque. None of the above studies have focused attention on the presence of eosinophils and this was probably based on an older study which has suggested that total white blood count is a better correlate of long-term than differentials [12]. Eosinophils, however, play an important role in inflammation and can identify the etiology of inflammation since they denote hypersensitivity inflammation [13-15]. Eosinophils are pleiotropic multifunctional leukocytes involved in initiation and propagation of inflammatory responses and thus have important roles in the pathogenesis of inflammatory diseases. These cells are also bone marrow-derived granulocytic leukocytes which express $\mathrm{H} 4$ histamine receptors in their surface. These receptors facilitate eosinophil chemotaxis toward mast cells which are the major producers of an array of inflammatory soluble mediators. These inflammatory mediators can induce the coronary hypersensitivity associated Kounis syndrome manifesting as vasospastic angina and/or acute myocardial 
infarction [13]. Soluble mediators secreted by mast cells and eosinophils modulate also reciprocal interactions between these two cells in the so called allergic effector unit [16]. Several studies have shown the role of eosnophils as a novel biomarker for risk stratification of patients with coronary artery disease.

In a recent study [17], it was found that eosinophil count in the third tertile was associated with an increased risk of all cause long-term mortality after the initial 6 months following percutaneous coronary intervention. This was attributed to an increased risk of thrombus formation because eosinophils infiltrate the site of stent implantation and release a number of mediators that can increase platelet activation and aggregation [18]. Indeed, stent thrombosis with thrombus infiltrated by eosinophis and/or mast cells constitutes the type III variant of Kounis syndrome [19].

In another study [20] the relationship between eosinophil count and coronary artery calcification in 1,363 consecutive participants with clinical suspicion of coronary heart disease was assessed. Evaluation of the relationship between coronary artery calcification score was determined by multislice computed tomography and peripheral eosinophil count as well as major cardiovascular risk factors, including age, body mass index, smoking status, hypertension, dyslipidemia, diabetes mellitus, high-sensitivity C-reactive protein and estimated glomerular filtration rate. Positive correlations were found between age and eosinophil count.

In a thrombus aspiration study of 209 consecutive patients [21] with acute coronary syndromes during emergency coronary angiography 165 thrombus sample sections were stained with hematoxylin and eosin and were examined for eosinophils. Eosinophil infiltration was observed predominantly in the area between white thrombus and red thrombus in 106 samples. The conclusion was that eosinophils may play an important role in coronary occlusion by promoting thrombus growth.

Furthermore, in a genome-wide association study [22] for sequence variants affecting eosinophil counts in blood it was found that a nonsynonymous single-nucleotide polymorphism at $12 \mathrm{q} 24$, in $\mathrm{SH} 2 \mathrm{~B} 3$, was associated significantly with myocardial infarction.

In conclusion, white blood count differentials constitute independent predictors of adverse outcomes following acute coronary syndromes and percutaneous coronary intervention. Eosinophil count in particular emerges also as novel biomarker for risk stratification on coronary artery patients. This is essential for elucidation of the etiology of inflammation because denotes hypersensitivity inflammation.

\section{Conflict of interest None.}

\section{References}

1. Mulvihill NT, Foley JB (2002) Inflammation in acute coronary syndromes. Heart 87:201-204

2. Quinaglia E Silva JC, Coelho-Filho OR et al (2013) Peri-infarct zone characterized by cardiac magnetic resonance imaging is directly associated with the inflammatory activity during acute phase myocardial infarction. Inflammation 2013 Nov 27. [Epub ahead of print]

3. Nozue T, Fukui K, Yamamoto S et al (2013) C-reactive protein and future cardiovascular events in statin-treated patients with angina pectoris: the extended TRUTH study. J Atheroscler Thromb 20:717-725

4. Palmerini T, Brener SJ, Genereux P et al (2013) Relation between white blood cell count and final infarct size in patients with STsegment elevation acute myocardial infarction undergoing primary percutaneous coronary intervention (from the INFUSE AMI Trial). Am J Cardiol 112:1860-1866

5. Palmerini T, Mehran R, Dangas G et al (2011) Impact of leukocyte count on mortality and bleeding in patients with myocardial infarction undergoing primary percutaneous coronary interventions: analysis from the harmonizing outcome with revascularization and stent in acute myocardial infarction trial. Circulation 123:2829-2837

6. Ndrepepa G, Braun S, Iijima R et al (2009) Total leucocyte count, but not $\mathrm{C}$-reactive protein, predicts 1 -year mortality in patients with acute coronary syndromes treated with percutaneous coronary intervention. Clin Sci (Lond) 116:651-658

7. Azab B, Zaher M, Weiserbs KF (2010) Usefulness of neutrophil to lymphocyte ratio in predicting short- and long-term mortality after non-ST-elevation myocardial infarction. Am J Cardiol 106:470-476

8. Yilmaz M, Tenekecioglu E, Arslan B et al (2013) White blood cell subtypes and neutrophil-lymphocyte ratio in prediction of coronary thrombus formation in non-st-segment elevated acute coronary syndrome. Clin Appl Thromb Hemost. 2013 Nov 6. [Epub ahead of print]

9. Williams BA, Merhige ME (2013) Association between neutrophil-lymphocyte ratio and impaired myocardial perfusion in patients with known or suspected coronary disease. Heart Lung 42:436-641

10. Akpek M, Kaya MG, Lam YY et al (2012) Relation of neutrophil/ lymphocyte ratio to coronary flow to in-hospital major adverse cardiac events in patients with ST-elevated myocardial infarction undergoing primary coronary intervention. Am J Cardiol 110: 621-627

11. Chung S, Song YB, Hahn JY et al (2013) Impact of white blood cell count on myocardial salvage, infarct size, and clinical outcomes in patients undergoing primary percutaneous coronary intervention for ST-segment elevationmyocardial infarction: a magnetic resonance imaging study. Int $\mathbf{J}$ Cardiovasc Imaging. 2013 Oct 9. [Epub ahead of print]

12. Gurm HS, Bhatt DL, Lincoff AM et al (2003) Impact of preprocedural white blood cell count on long term mortality after percutaneous coronary intervention: insights from the EPIC, EPILOG, and EPISTENT trials. Heart 89:1200-1204

13. Kounis NG (2013) Coronary hypersensitivity disorder: the Kounis syndrome. Clin Ther 35:563-571

14. Kounis NG, Mazarakis A, Tsigkas G, Giannopoulos S, Goudevenos J (2011) Kounis syndrome: a new twist on an old disease. Future Cardiol 7:805-824

15. Kounis NG (2006) Kounis syndrome (allergic angina and allergic myocardial infarction): a natural paradigm? Int J Cardiol 110:7-14 
16. Minai-Fleminger Y, Levi-Schaffer F (2009) Mast cells and eosinophils: the two key effector cells in allergic inflammation. Inflamm Res 58:631-638

17. Toor IS, Jaumdally R, Lip GY, Millane T, Varma C (2012) Eosinophil count predicts mortality following percutaneous coronary intervention. Thromb Res 130:607-611

18. Rohrbach MS, Wheatley CL, Slifman NR, Gleich GJ (1990) Activation of platelets by eosinophil granule proteins. J Exp Med 172:1271-1274

19. Biteker M (2010) A new classification of Kounis syndrome. Int J Cardiol 145:553
20. Tanaka M, Fukui M, Tomiyasu K et al (2012) Eosinophil count is positively correlated with coronary artery calcification. Hypertens Res 35:325-328

21. Sakai T, Inoue S, Matsuyama TA (2009) Eosinophils may be involved in thrombus growth in acute coronary syndrome. Int Heart J 50:267-277

22. Gudjartsson DF, Bjornsdottir US, Halapi E et al (2009) Sequence variants affecting eosinophil numbers associate with asthma and myocardial infarction. Nat Genet 41:342-347 